\title{
Was Fachkräfte wissen müssen
}

\author{
Der Qualifikationsrahmen Soziale Arbeit
}

Peter Buttner und Ulrich Bartosch

Ende Mai 2006 hat der Fachbereichstag Soziale Arbeit den "Qualifikationsrahmen Soziale Arbeit « (QR SArb) beschlossen. Damit ist die Soziale Arbeit das erste Studienfach in Deutschland, das sich in Bezug auf die europäische Ausbildungslandschaft mit einem fachspezifischen Qualifikationsrabmen positioniert hat und Studierenden in Bezug auf die Qualifikationsniveaus Bachelor und Master eine allgemeine Orientierung über die fachspezifischen Qualifizierungsanforderungen gibt.

Mit der Entwicklung eines fachspezifischen Qualifikationsrahmens hat die Soziale Arbeit in einer Zeit zunehmender Entgrenzung der Fachdisziplinen einen Schritt zu ihrer Konsolidierung getan und den Boden für eine strukturelle Verbesserung der beruflichen Einmündung sowie der Promotionsmöglichkeiten für Absolventinnen und Absolventen der Sozialen Arbeit gefestigt.

Das Ziel der Qualifikationsrahmen ist, mit einer breiten und differenzierten Beschreibung, das europäische Bildungssystem bis in seine Verästelungen durchsichtig, verständlich und vergleichbar zu machen und somit auch die Orientierung und Mobilität der Studierenden zu fördern. Der Anstoß zur Erstellung fachspezifischer Qualifikationsrahmen kam in Deutschland durch den (nationalen) Qualifikationsrahmen für die Hochschulen (HRK \& KMK, 2005), der selbst wiederum in Beziehung steht zu dem - in Erstellung befindlichen - europäischen Qualifikationsrahmen für Hochschulabschlüsse.

Prof. Dr. Peter Buttner (Fachhochschule München) und Prof. Dr. Ulrich Bartosch (Katholische Universität EichstättIngolstadt) sind Vorstandsmitglieder des Fachbereichstages Soziale Arbeit der Hochschulrektorenkonferenz.

E-Mail ulrich.bartosch@ku-eichstaett.de
Der nationale Qualifikationsrahmen beschreibt allgemein und für alle Studienfächer (1), welche Qualifikationsmerkmale den drei akademischen Niveaustufen Bachelor, Master und Doktorat zukommen, welche Lernergebnisse vorgesehen sind, die angestrebten Kompetenzen und die formalen Aspekte der Ausbildungslevels. Nach einer intensiven Arbeitsphase hat der Fachbereichstag unter breiter Einbeziehung der Fachbereiche und Fakultäten für Soziale Arbeit am 31. Mai 2006 in Berlin den (fachspezifischen) »Qualifikationsrahmen Soziale Arbeit « (QR SArb) beschlossen. Verantwortlich für diesen Prozess war Ulrich Bartosch mit der von ihm gebildeten Arbeitsgruppe. (2)

Der Qualifikationsrahmen Soziale Arbeit leitet sich aus dem nationalen Qualifikationsrahmen ab, übernimmt die wesentlichen Abgrenzungsmerkmale zwischen Bachelor und Master, findet aber zu einer eigenständigen Darstellung der Qualifikationen. Praxisbezogene und wissenschaftliche Qualifikation spielen eine wichtige Rolle, jedoch nicht als Antipoden, vielmehr im Sinne einer notwendigen Ergänzung. Abgesehen davon, dass auch wissenschaftliche Tätigkeit als eine Praxis aufgefasst werden kann, unterliegt dem Qualifikationsrahmen Soziale Arbeit der Gedanke, dass jeder Bachelorstudiengang notwendigerweise auch für das wissenschaftsorientierte Weiterstudieren (Master) qualifizieren muss - unabhängig davon, dass der Bachelor als erster berufsqualifizierender Abschluss gilt.

Der Qualifikationsrahmen Soziale Arbeit teilt sich in sieben Abschnitte. Jeder dieser Abschnitte ist in drei Teile gegliedert: einer allgemeinen Beschreibung und je einer Darlegung des Bachelor- und des Master-Levels. Die Folge der Abschnitte entspricht einer prozessualen Logik: Durch Wissen und Verstehen (A) erwächst aus den (an die Soziale Arbeit herangetragenen) Aufgabenstellungen ein Aufgaben- bzw. Problemverständnis.
Mittels Beschreibung, Analyse und Bewertung (B) kann eine Aufgabenbeschreibung erfolgen, die in die Planung und Konzeption (C) Sozialer Arbeit mündet. So entstandene Konzepte bedürfen gleichwohl weiterer Recherche (D) und ggf. Forschung. Schließlich muss das Handeln der Sozialen Arbeit bzw. organisiert (E) und evaluiert (E) werden. Im Qualifikationsrahmen Soziale Arbeit schließen sich ein Abschnitt F (allgemeine professionelle Fähigkeiten) und G (Haltungen) an.

Als Grundlage der Qualifikation in der Sozialen Arbeit setzt der Qualifikationsrahmen Soziale Arbeit das Wissen und Verstehen. Absolventinnen und Absolventen der Sozialen Arbeit verfügen »über grundlegendes, sicheres Wissen und Verständnis der theoretischen und angewandten Sozialarbeitswissenschaften sowie mindestens der relevanten Wissensbestände der korrespondierenden Wissenschaftsbereiche «. Gegenüber den Bachelor verfügen Master der Sozialen Arbeit über weiter- und tiefer-gehende Qualifikationen: Ihr Wissen und Verständnis ist umfassend und schließt ausgewählte Methoden qualitativer und quantitativer Sozialforschung ein. Ihre Theorie-, Modell- und Methodenkenntnisse sind vertieft. Sie haben darüber hinaus einen vertieften Überblick über die aktuelle nationale und internationale Forschung und Entwicklung in der Sozialen Arbeit.

Im Teil B (Beschreibung, Analyse und Bewertung) geht es um das Erkennen von Aufgabenstellungen in der Sozialen Arbeit und ihre Zuordnung zu definierten Aufgaben- bzw. Problemfeldern. Während Bachelor in erster Linie Aufgabenstellungen zu bewältigen haben, die als typisch einzustufen sind, haben die Master die Fähigkeit, auch neue und ungewöhnliche diagnostische Aufgabenstellungen zu bearbeiten und können Kolleginnen und Kollegen bei der Analyse derselben fachlich anleiten. 
Bei Abschnitt C (Planung und Konzeption) geht es nicht nur um die Vorbereitung von Interventionen (»Dienstleistungen «), sondern auch von Hilfesystemen und um die Prozessplanung. Auch hier unterscheiden sich Bachelor und Master durch den Grad der Eigenständigkeit und hinsichtlich der Fähigkeit, Lösungsstrategien für neue und unbekannte Aufgabenstellungen zu entwickeln.

Der Abschnitt D (»Recherche und Forschung in der Sozialen Arbeit «) hat die deutlichste Wissenschaftsorientierung, denn es geht für Bachelor und Master um die Bearbeitung von Forschungsfragen und um fachliche Informationsbeschaffung. Master werden breitere Kenntnisse und weit mehr Eigenständigkeit als dem Bachelor attestiert Während Bachelor beispielsweise die Fähigkeit zugeordnet ist, angeleitete Praxisforschung zu betreiben, verfügen Master über die Fähigkeit, das Design von Untersuchungen zu entwickeln, Forschungsergebnisse kritisch zu bewerten und innovative Methoden und Strategien zu entwickeln.

\section{Das Format mit Inhalt füllen}

Die Deutsche Gesellschaft für Soziale Arbeit versteht sich als unabhängiges Forum für die Wissenschaft und Praxis Sozialer Arbeit. Sie nimmt für sich in Anspruch, die Sache der Profession der Sozialen Arbeit und die Disziplin der Sozialarbeitswissenschaft zu vertreten. Ihr Vorsitzender Prof. Dr. Wolf Rainer Wendt äußert sich in einem Kurzinterview zu dem im letzten Jahr verabschiedeten Qualifikationsrahmen Soziale Arbeit.

Blätter der Wohlfahrtspflege: Wie steht die Deutsche Gesellschaft für Soziale Arbeit zu dem Qualifikationsrahmen des Fachbereichstages?

Wolf Rainer Wendt: Unsere Gesellschaft begrüßt diese Ausarbeitung. Wichtig ist die grundsätzliche Entscheidung für ein generalistisches Studium. Die Darstellung kommt einem wissenschaftlichen Anspruch nach, den die Soziale Arbeit wie andere Disziplinen in der Lehre an Hochschulen erheben kann und formuliert diesen Anspruch auf den Qualifikationsniveaus des Bachelor- und des Masterstudiums. Das Format, das den Studiengängen zugeschrieben wird, bedarf aber einer inhaltlichen Ausfüllung. Im Text wird von einer »Prozesslogik « Sozialer Arbeit sowie von einem »kollektiven Wissens- und Fähigkeitskanon " gesprochen - ohne nähere Angaben zur Sache.

Blätter der Wohlfahrtspflege: Welcher Inhalt passt Ihrer Ansicht nach in den Qualifikationsrahmen?

Wolf Rainer Wendt: Wir haben zur inhaltlichen Ausgestaltung des Studiums der Sozialen Arbeit ein Kerncurriculum zur Diskussion gestellt (nachzulesen auf unserer Website http://www.dgsinfo.de, Rubrik Mitteilungen, Online-Mitteilung 77). Die Hochschulausbildung muss den ganzen Gegenstandsbereich der Sozialen Arbeit auf dem Bachelor-Level behandeln. Die Berufsbefähigung, die ein Bachelor haben sollte, hängt weniger von der Fülle des Wissens aus herkömmlichen »Bezugsdisziplinen « als von der Kompetenz ab, die in der Behandlung der Problematik gesellschaftlicher Daseinsgestaltung und individueller Lebensführung in konkreten personen- und situationsbezogenen Aufgabenstellungen erworben wird. Einen breiten Einblick in die Praxis eingeschlossen. Das Masterstudium baut auf dieser Fundierung, aber nicht durch »Mehr vom Gleichen «, sondern mit der Kompetenz für Entwicklungs- und Steuerungsaufgaben.

Blätter der Wohlfahrtspflege: Wie müssen nun die nächsten Schritte aussehen? Wolf Rainer Wendt: Zu klären ist die Differenzierung der Masterstudiengänge. Die Profession braucht einerseits einen sozialarbeitswissenschaftlich akzentuierten, stärker forschungsorientierten konsekutiven Masterstudiengang, der für Leitungsaufgaben qualifiziert und der (in Verbindung mit oder an einer Universität) die Voraussetzungen für eine Promotion schafft und so in Zukunft eine Selbstrekrutierung der Lehre im Fachgebiet ermöglicht. Andererseits ist darauf hinzuwirken, dass für die Spezialisierungen im Handlungsfeld solche anwendungsbezogenen Studiengänge vorhanden sind, die für eine Fachsozialarbeit qualifizieren, damit der Profession zugeordnet bleiben und nicht den Zusammenhang mit ihr verlieren.

Der Abschnitt E (»Organisation, Durchführung und Evaluation in der Sozialen Arbeit «) behandelt die sichtbarste Ebene der Sozialen Arbeit: von ihrer Organisation und Durchführung bis hin zu ihrer Evaluation: Einschätzung und Einsatz sächlicher und personeller Ressourcen. Für Master wird u. a. festgestellt, dass sie auch die Weiterentwikklung von Methoden und Qualitätsmanagementsystemen betreiben können.

Der Abschnitt F schließlich (»Professionelle allgemeine Fähigkeiten und Haltungen in der Sozialen Arbeit «) benennt » weitere, nicht fachspezifische Fähigkeiten, die für die erfolgreiche, professionelle Soziale Arbeit als Vorbedingung gelten müssen «. Der Abschnitt G (»Persönlichkeit und Haltungen «) differenziert bewusst nicht zwischen Bachelor und Master.

\section{Fazit und Ausblick}

Der Qualifikationsrahmen Soziale Arbeit ist - nach Modularisierung und Umstellung auf Bachelor und Master - eine weitere Mitwirkung der Fachbereiche der Sozialen Arbeit an der Harmonisierung des europäischen Hochschulraumes und der Abstimmung von hochschulisch und außerhochschulisch erworbenen beruflichen Qualifikationen.

Der Qualifikationsrahmen Soziale Arbeit ist kein berufspolitisches Dokument. Man könnte ihn als Ausbildungsversprechen bezeichnen, das den Mindestumfang der mit dem Abschluss Bachelor oder Master erworbenen und geprüften Qualifikationen umfasst. Er trifft keine inhaltliche Festlegung und stellt keine endgültige und vollständige Liste einzelner professioneller Kompetenzen vor. Im Wettstreit der Disziplinen kann er der Sozialen Arbeit als legitimatorisches Instrument dienen - beispielsweise um gute $\mathrm{Zu}$ gangsmöglichkeiten zur Promotion.

Die Anregungen und Kommentare, die bis hin zur Abschlussdiskussion vorgelegt und behandelt wurden, haben wesentlich zur Qualität des Qualifikationsrahmens Soziale Arbeit beigetragen. In der einstimmigen (!) Beschlussfassung wurde eine Revision des Qualifikationsrahmens Soziale Arbeit in eineinhalb Jahren vorgesehen. Eine fraglos wichtige Weiterentwicklung wäre die Hinzunahme der Doktoratsebene. Diskutiert wird auch, wie die Anschlussstellen an internationale Doku- 
mente der Sozialen Arbeit prägnanter herausgearbeitet werden können.

\section{Anmerkungen}

(1) Auch ungeachtet der Hochschultypen (z. B. Fachhochschulen vs. Universitäten) und der Wissenschaftsgebiete ( $\mathrm{z}$. B. Geisteswissenschaften und Naturwissenschaften).

(2) M.A. Dipl.-Soz.-Päd Christine Speth, zunächst HRK, Bonn, jetzt Universität Eichstätt und M.A. Dipl.-Soz.Päd Anita Maile, Bologna-Beraterin FH-Frankfurt am Main.

\section{Grundsicherung für Arbeitsuchende}

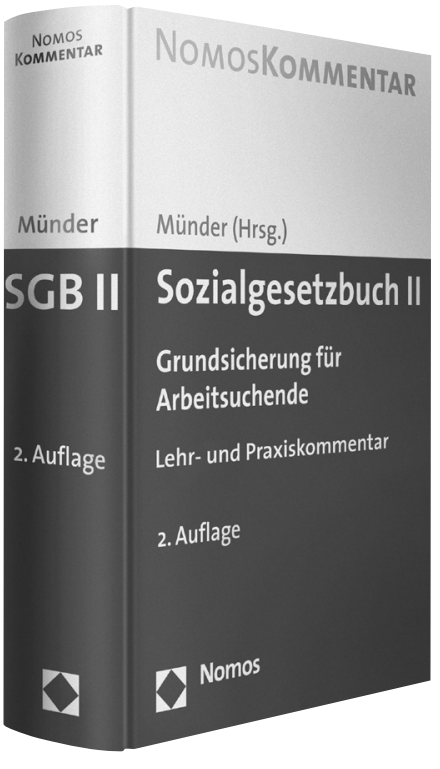

\section{Sozialgesetzbuch II}

Grundsicherung für Arbeitsuchende Lehr- und Praxiskommentar Herausgegeben von Prof. Dr. Johannes Münder, TU Berlin 2. Auflage 2007, 786 S., geb., 44,- $€$, ISBN 978-3-8329-1783-8

\section{Literatur}

HRK/KMK (2005): Qualifikationsrahmen für Hochschulabschlüsse. Im Internet unter: http://www.kmk.org/doc/ beschl/BS_050421_Qualifikationsrahmen_AS_Ka.pdf\#search=\%22hrk\%20q ualifikationsrahmen $\% 20 \% 22$.

Bartosch U. u. a. [Arbeitsgruppe Qualifikationsrahmen Soziale Arbeit des Fachbereichstages Soziale Arbeit] (2006): Qualifikationsrahmen Soziale Arbeit (QR SArb), Version 4.0 (bislang als Broschüre über den Fachbereichstag Soziale Arbeit zu beziehen und im Internet unter http://www.fbts.de abzurufen).
Die Grundsicherung für Arbeitsuchende ist in wesentlichen Punkten novelliert. Durch das SGB II-Änderungsgesetz im April und insbesondere durch das „Fortentwicklungsgesetz" sind weitgehende Leistungseinschnitte zum 1. August 2006 Gesetz geworden.

Was bedeuten diese mit der „Optimierung des Leistungsrechts“ und der „Vermeidung von Leistungsmissbrauch“ begründeten Änderungen für das Regelungssystem des SGB II und die Auslegungspraxis?

Die Neuauflage des LPK gibt unmittelbar nach der Reform die praktischen Antworten. Der LPK-SGB II

- bietet eine vollständige, verständliche und topaktuelle Kommentierung des novellierten SGB II, unter besonderer Berücksichtigung der Praxisauswirkung insbesondere der Leistungskürzungen,

- behandelt dabei ausführlich die Erweiterung der Bedarfsgemeinschaft um Jugendliche, die das 25 . Lebensjahr noch nicht vollendet haben, zusammen mit den dazugehörenden Beweislastfragen und

- interpretiert kritisch die Verschärfung von Sanktionsmöglichkeiten.

Die für die Auslegung des Gesetzes - vor allem für die Interpretation der vielfältigen unbestimmten Rechtsbegriffe - prägenden Entscheidungen der Sozialgerichte seit In-Kraft-Treten des SGB II sind durchgängig berücksichtigt.

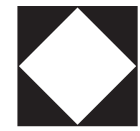

Nomos 